\title{
Quality Improvement (QI): A Splendid Driver for Achieving the Third 90 in Addis Ababa, Ethiopia
}

\author{
Abay Sisay ${ }^{1 *}$, Bekelech Bayou ${ }^{2}$ and Abrham Tesfaye ${ }^{3}$ \\ ${ }^{1}$ Department of Medical Laboratory Science, College of Health Science, Addis Ababa University, Ethiopia \\ ${ }^{2}$ USAID/JSI Strengthening Ethiopian's Urban Health Program (SEUHP), Addis Ababa, Ethiopia
}

${ }^{3}$ Addis Ababa Public Health Research and Emergency Management, Addis Ababa, Ethiopia

\begin{abstract}
Background: Routine HIV viral load tests improve treatment quality and individual health outcomes for people living with HIV. Quality improvement (QI) consists of systematic and continuous actions that lead to measurable improvement in health care services. Thus, the aim of this study was to assess the effect of Quality Improvement (QI) on achieving routine HIV viral load implementation in Addis Ababa, Ethiopia.
\end{abstract}

Methods: Facility based prospective follow up study design approach was used from January 2016 to December 2016. Data was entered, cleaned using EPI-Data 3.1 and exported to SPSS version 20 software for further processing and analysis. The effect of QI was measured and monitored by test change using run chart.

Results: At the end of the quality improvement, the monthly routine viral load performance samples using one analyzer were 3255 from less than 676 at baseline. The routine viral load feedback arrival within agreed turnaround time and regularly archival availability increase from 6 health facilities $(7.2 \%)$ to $65 \%$ at 54 health facilities by December 2016, from the 83 ART monitoring health facilities sent their HIV viral load sample. Implementation of continuous quality improvement is a game changer in transforming laboratory quality and positively impact on meeting the third 90 in Addis Ababa, Ethiopia.

Conclusion: The QI model which have implemented at Addis Ababa health research HIV viral load laboratory achieve and worth good experiences with a range of benefits. We strongly believe that healthcare has much to gain by successfully implementing Quality improvement principles within their routine programs.

Keywords: Addis Ababa; HIV; QIP; Viral load

\section{Background}

Since the first Acquired Immune Deficiency Syndrome (AIDS) cases identified in 1986, Ethiopia had started to respond to the epidemic in different approaches [1]. Aligning with a global fast track response to ending AIDS epidemic by the year 2030 achieving triple 90 s is UNAIDS motivated plan to be achieved as of 2020. In resource limited countries, antiretroviral therapy (ART) treatment failure and patient monitoring system dependent on clinic-immunological methods, though they lack sensitivity/specificity to detect virological failures of ART, which is the gold standard for ART treatment failure [2]. Though, because of financial (40-85 USD per test) and operational barriers, while recognizing viral load as the gold standard for ART monitoring, focused on using clinical and immunological criteria for monitoring ART in developing countries [3]

The current WHO and Ethiopian national consolidated guidelines recommend Viral Load (VL) testing as the preferred monitoring tool for diagnosing and confirming the failure of ART and hence, there is a need to create access to high quality routine VL testing through efficient specimen referral network, with limited reliance on laboratory monitoring [4].

Quality Improvement (QI) strives to enable an organization to achieve the ideal critical pathway, which is one that allows the health care provider team and patient to interact productively and efficiently to achieve optimal health outcomes. It has a green light of forwarding achievement [5]. Routine Human Immunodeficiency Virus (HIV) viral load tests improve treatment quality and individual health outcomes for people living with HIV, contribute to prevention and potentially reduce resource needs for costly second and third line HIV medicines [6].
Ethiopia has demonstrated remarkable progress in expanding HIV/ AIDS care quality of health care in terms of improving patient safety, effectiveness and patient-centeredness. Despite this progress, substantial coverage and inequalities still exist in routine HIV viral load monitoring [7]. As part of this execution plan, achieving the third 90, capacity buildings seize the crucial part. Accordingly, Sensitization workshop on routine HIV viral load was conducted for laboratory personnel, health care providers and program management team at different time by Addis Ababa. Following the training on January 2016 the routine viral load service was initiated by the available VL analyzer at AAHRL, right away the recommendation of UNAIDS shifting from targeted viral load management, using existing sample referral.

As indicated in Figure 1, the quality improvement implementation frame work following the national health model, QI had been implemented at all level.

According to the annual report of the Addis Ababa City Administration Health Bureau (AACAHB) for July 2015, there were a total of 125,994 HIV positive clients (94.4\% adults) who enrolled

*Corresponding author: Abay Sisay, Department of Medical Laboratory Science, College of Health Sciences, Addis Ababa University, Addis Ababa, Ethiopia, Tel: 251911547032; E-mail: abusis27@gmail.com

Received March 23, 2018; Accepted April 10, 2018; Published April 17, 2018

Citation: Sisay A, Bayou B, Tesfaye A (2018) Quality Improvement (QI): A Splendid Driver for Achieving the Third 90 in Addis Ababa, Ethiopia. J AIDS Clin Res 9: 765 doi: 10.4172/2155-6113.1000765

Copyright: @ 2018 Sisay A, et al. This is an open-access article distributed under the terms of the Creative Commons Attribution License, which permits unrestricted use, distribution, and reproduction in any medium, provided the original author and source are credited. 


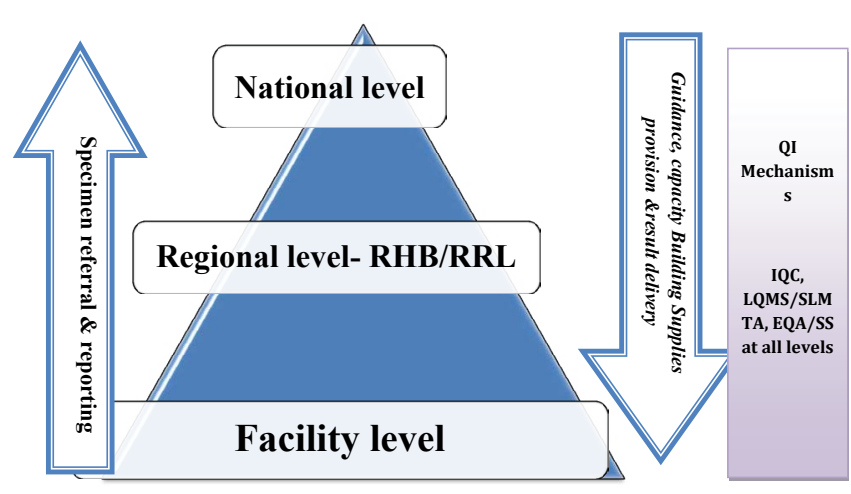

Figure 1: Quality improvement implementation frame work on clinical laboratory in Addis Ababa, Ethiopia.

to ART services, among which 74,986 ( $94.8 \%$ adults) had started ART and there were 53,677 (95.5\% adults) current on ART. However, the HIV viral load performance were 966 per annum, which is far from the UNAIDS recommendation. Hence, the regional HIV viral load monitoring laboratory, aggressively take a plan kicking off a Quality Improvement (QI) in order to transform and take off the low performance of viral load test by proffer a proven methodology for improving its performance [8]. Hence, this study intends to assess the effect of Quality Improvement (QI) on achieving routine HIV viral load implementation in Addis Ababa, Ethiopia and this will help policy makers and the scientific community for further programmatic and research endeavor.

\section{Methodology}

\section{Study design and setting}

Facility based prospective follow up study design approach was used from January to December 2016 in Addis Ababa Health research laboratory (AAHRL). Addis Ababa is the capital city of Ethiopia, which is located at the heart of the country with the area of about $540 \mathrm{~km}^{2}$. It is the biggest city in the country and a chartered city having three layers of government namely, city government at the top, 10 sub cities in the middle and 116 woredas, with population of 2, 854, 462 [9]. There are 6 regional, 5 federal (including one university hospital), 2 NGO-supported, 30 private, 1 defense, 1 prison and 1 police hospitals laboratories. There are also 100 (currently functional) public and 4 NGO-supported health centers laboratories, 7 publics, 500 private and 31 NGO supported clinics laboratories and there is one accredited regional reference laboratory, Addis Ababa health research laboratory. As of 2015 report; under AACAHB, 111 health facilities were provided ART service (11 public hospitals, 77 government health centers, 14 private hospitals and 9 non-government clinics). Among them 83 Health facilities sent their HIV viral load sample to AAHRL, through Ethiopian postal agency referral linkage $[10,11]$. This laboratory is the only government laboratory providing viral load service to the government ART serving health facilities. The present study was conducted at Addis Ababa Health research laboratory.

\section{Key strategies which accomplished}

Base line assessment was conducted to know the status of routine VL performance and its contribution for improving the quality of care and services, which were the laboratory, perform a targeted approach. The laboratory shift its service directly from targeted to routine viral load monitoring approach following fast track UNAIDS recommendation of

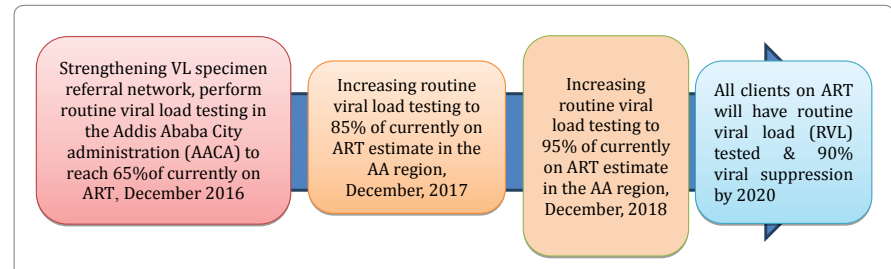

Figure 2: A phase based approach strategy implemented to routine HIV viral load testing, 2016-2020. Addis Ababa, Ethiopia.

the triple 90s. At this time there is low number of clients monitored and managed by a common QI tools like fishbone diagram used to identify quality problems and also utilize a driver diagrams to systematically identify interventions by using a prioritizing matrix's to address quality challenges as indicated (Figure 2). Moreover, to keep and meet the right path of the third 90 of UNAIDS recommendation, considering the time frame of 2016-2020 a phase based approach strategy had been implemented to routine HIV viral load testing by capacitating the current available viral load testing in Addis Ababa.

\section{Data quality control}

Before the implementation of the quality improvement, base line was performed to know the status of the viral load implementation and training was given to laboratory personnel who engaged on sample referral, ART clinical providers accordingly, by regional laboratory experts and by principal investigators. There was a regular control of data capturing quality by the principal investigators in order to maintain the data quality. Moreover, the Addis Ababa Health research laboratory is ISO 15189 accredited laboratory for the scope of Hematology, EID and GeneXpert, which have relatively good data quality management system [12].

\section{Data analysis}

The data for this study was gathered using structured checklist and observational questionnaire. Data was entered, cleaned using excel sheet and EPI-Data 3.1, then exported to SPSS version 20, software for further processing and analysis. Determinate factors were dissecting and fine to get the root cause for the low performance of routine viral load by using fishbone diagram and prioritizing matrix. The effect of QI implementation on routine viral load was measured and monitored by test change using run chart.

\section{Results}

The monthly routine viral load test performance using one analyzer were 3255 , which is around five-fold from the base line (676) as indicated in Table 1, Implementation of continuous quality improvement impact positively and a promising agenda on meeting the third 90 in Addis Ababa, Ethiopia, as indicate at the run chart in Figure 3 brings a significant change resulted in routine HIV viral load improvement.

QIP effect on routine viral load implementation in order to see the result by doing the baseline assessment and doing the problem via QI tool, then and monitor the progress using ran chart. Without altering the routine and using the established systems, the regional health bureau set an assertive but also achievable plan of routine viral load testing in the Addis Ababa to achieve the $3^{\text {rd }} 90$ target by the end of 2019 , which means $90 \%$ of clients will have their routine VL testing service and out of which $90 \%$ will have viral RNA copy of less than $1000 / \mathrm{ml}$ of plasma. This plan urge us to feel out of the box, to realize this pushy plan, and it was the time to inject a quality improvement in 
the system, Quality Improvement (QI) put forwards a proven tactic for improving routine HIV viral load in Addis Ababa.

As indicate in Figure 4 different actions were in use in order to drive the routine HIV viral load in a steadily manner, like sensitizing all clinical providers, a continuous monitoring of a quality indicators interpret the variation and measure progress of change quality improvement initiatives. As indicated in the graph, there was up and downs on performance, cause there were erratic and shortage of fine tips, chronic stock out of test tubes (EDTA, PPT, Nuctube) for sample collection at collection site of referring health facilities and there were equipment down time because of stock out of method calibrator at the testing laboratory. From this any one conclude as there were many existing challenges, even though there was good performance in the region. The reference regional laboratory manage it, by designing different approach like arranging extra payment duty for additional duty time, facilitating on site and on job training to capacitate health professionals working on Viral load analyzer, strengthening the laboratory backup system, timely feedback delivery system established, deployed laboratory mentor at sub city level by designing a structure to improve health professionals knowledge through regular mentorship, guide lines and provider support tools developed, oriented and distributed at health facility level. A promising change was observed on the referring health facilities, at the QI implementation there were appropriate RVL folder system for appropriate feedback archival, PSTs,

\begin{tabular}{|l|c|c|c|}
\hline Serial \# & Moth & $\begin{array}{c}\text { \# of routine viral load sample } \\
\text { run/one analyzer }\end{array}$ & Remark \\
\hline 1 & January, 2016 & 676 & \\
\hline 2 & February, 2016 & 1503 & \\
\hline 3 & March, 2016 & 1502 & \\
\hline 4 & April, 2016 & 1430 & \\
\hline 5 & May, 2016 & 1543 & \\
\hline 6 & June, 2016 & 2333 & \\
\hline 7 & July, 2016 & 877 & \\
\hline 8 & August, 2016 & 1485 & \\
\hline 9 & September, 2016 & 1136 & \\
\hline 10 & October, 2016 & 1320 & \\
\hline 11 & November, 2016 & 2432 & \\
\hline 12 & December, 2016 & $\mathbf{1 9 4 9 2}$ & \\
\hline & 2016 total & & \\
\hline
\end{tabular}

Table 1: Summary monthly performance of routine HIV viral load performance using one analyzer in Addis Ababa, Ethiopia, 2016. tracking log books available and archive accordingly, requested samples were return back to the referring health facilities within the agreed TAT and they were reviewed and archived it by the respective quality mangers. The other remarkable change were observed in the case of laboratory reagents, supplies and consumables by monitoring and availing at the head of time by implementing integrated pharmaceutical supply chain management system and regularly updating their bin cards, it makes the viral load analyzer regular functionality.

The RVL feedback arrival within agreed TAT and regularly archival availability increase from 6 health facilities $(7.2 \%)$ to $65 \%$ at 54 health facilities by Dec. 2016 from the 83 ART monitoring health facilities sent their HIV viral load sample to AAHRL as depict in Table 2.

Based on the evidence that obtained from the base line assessment, different action points set and activities were prioritized according to their importance to improve the quality of care and service by a phased approach. Activities that were conducted and used as driving factors to quality improvement were; sensitization workshop organized for managers at different level which are responsible for the work. Presenting of the base line assessment were part of the sensitization workshop and proposed corrective actions from the audience were one of the value add for the barriers. The other activity conducted was

\section{\begin{tabular}{|l|l|l|}
\hline Variable & Baseline & At the end of Q
\end{tabular}}

\begin{tabular}{|l|l|l|l|}
\hline Frequency & Percentage (\%) & Frequency & Percentage (\%)
\end{tabular}

Health facilities with Routine Viral Load feedback archived in a separate folder

\begin{tabular}{|l|c|c|c|c|}
\hline Yes & 6 & 7.2 & 54 & 65 \\
\hline
\end{tabular}

\begin{tabular}{|l|l|l|l|l|}
\hline No & 77 & 92.8 & 29 & 35 \\
\hline
\end{tabular}

Health facilities received their Routine Viral Load result within agreed TAT

\begin{tabular}{|l|l|l|l|l|}
\hline Yes & 6 & 7.2 & 54 & 65 \\
\hline
\end{tabular}

\begin{tabular}{|l|l|l|l|l|}
\hline No & 77 & 92.8 & 29 & 35 \\
\hline
\end{tabular}

Health facilities with assigned Routine Viral Load focal person

\begin{tabular}{|l|c|c|c|c|}
\hline Yes & 11 & 13 & 79 & 95 \\
\hline
\end{tabular}

\begin{tabular}{|l|l|l|l|l|} 
No & 72 & 87 & 4 & 5 \\
\hline
\end{tabular}

Health facilities with Routine Viral Load PSTs, log books, tracking sheets availability and utilization

\begin{tabular}{|l|c|c|c|c|}
\hline Yes & 21 & 25 & 82 & 99 \\
\hline No & 62 & 75 & 1 & 1 \\
\hline \multicolumn{5}{|l|}{ Health facilities assessed and mentored by Lab mentors } \\
\hline Yes & 5 & 6 & 63 & 76 \\
\hline No & 78 & 94 & 20 & 24 \\
\hline
\end{tabular}

Table 2: Progress of health facilities from the baseline after the Implementation of QI in Addis Ababa, Ethiopia, $2016(n=83)$

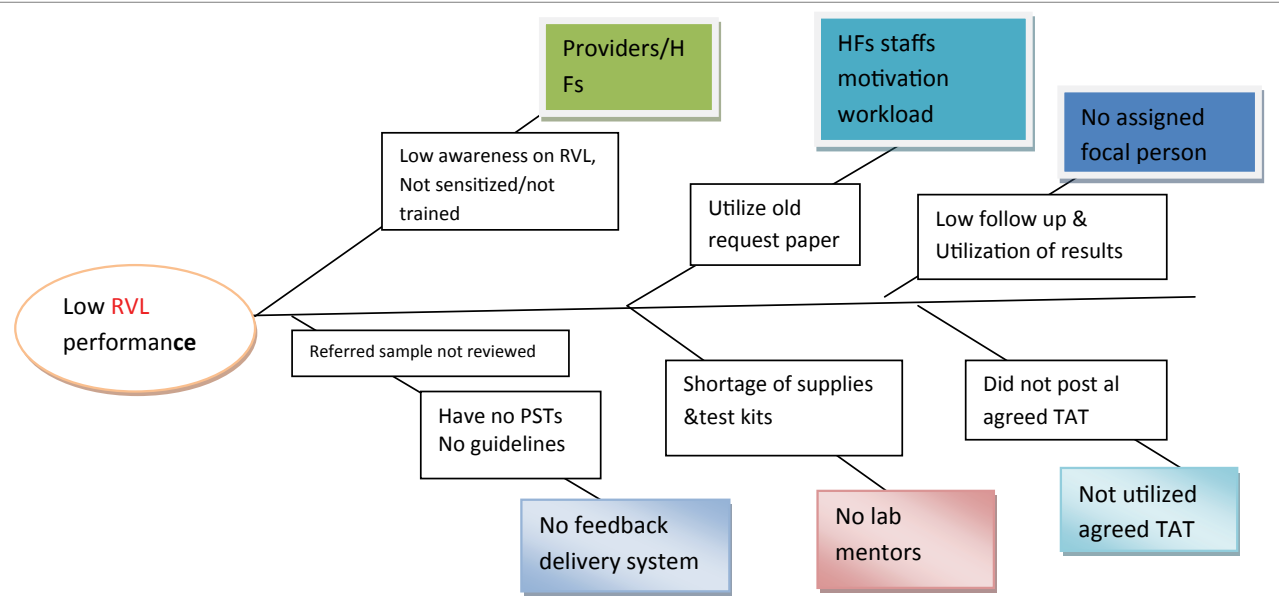

Figure 3: Fishbone diagram indicate the probable cause of the low routine viral load performance in Addis Ababa, Ethiopia, 2016. 


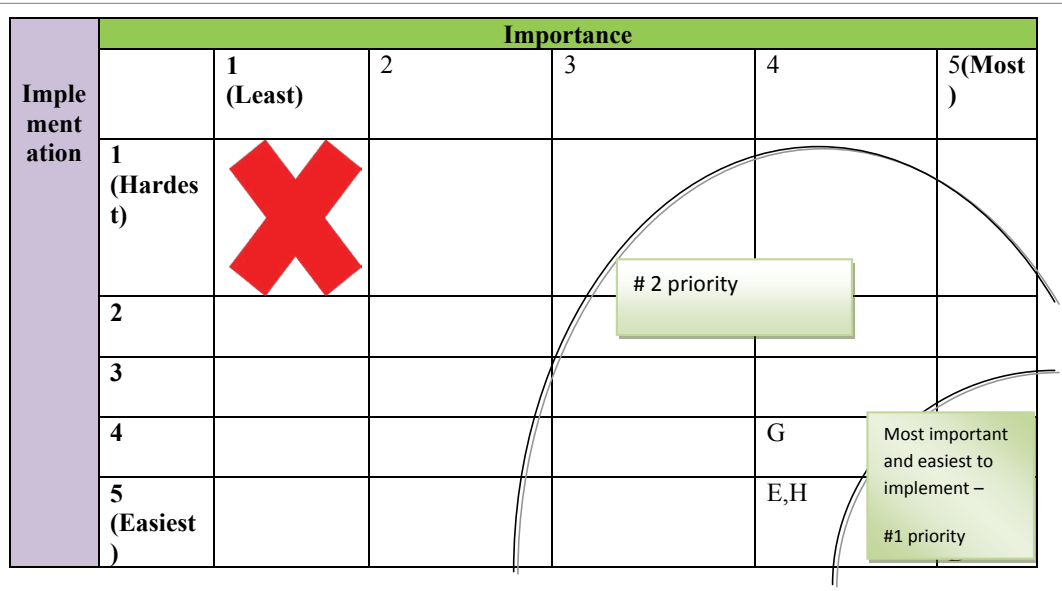

QI Tool: Focusing Matrix was performed to give the prioritizing the most important and easiest to implement from the computing factors for the low performance of routine HIV viral load performance

A. Ensure appropriate RVL folder system for appropriate feedback archival, PSTs, tracking $\log$ books available and archive accordingly.

B. Support the recording and analysis of requested samples to know when they return back at the facility level.

C. Support and encourage the HFs to assign a designated VL focal person.

D. Support the postal man to deliver the result to the concerned body and kept the record @ ART clinics.

E. Conduct mentorship on Preparation of action plan for unreturned results and the root cause analysis.

F. Conduct site assessment regularly to scale up the RVLM and maintaining the TAT.

G. Follow the stock/supplies level and analyzer functionality regularly.

H. Train the lab personnel and clinical providers on the routine VL scale up and feedback arrival within agreed TAT and utilization of results.

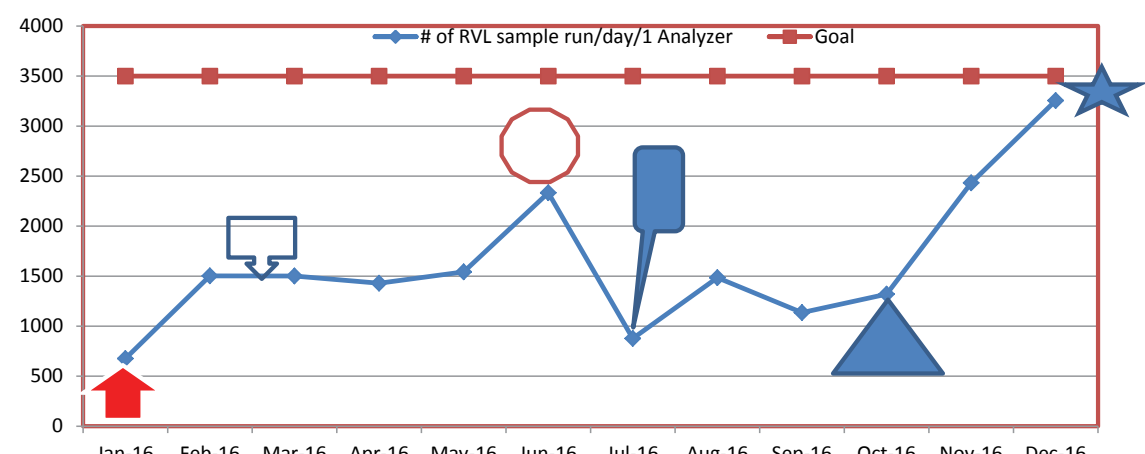

Jan-16 Feb-16 Mar-16 Apr-16 May-16 Jun-16 Jul-16 Aug-16 Sep-16 Oct-16 Nov-16 Dec-16

Baseline assessment conducted $\Gamma=$ faced a stock out in a national manner

$\square$ : Quality Indicators Monitored; $\triangle$ :Installed Additional Analyzer, Trained

Additional Lab Personnel, Additional Postal Office Personnel Evolved; C: All Clinicians

are being Sensitized on VL Service Utilization; 25 : Make Sustainability and Make It Routine, then After

Figure 4: Run chart depicting the effect of QI on routine HIV viral load in Addis Ababa, Ethiopia.2016.

organizing of sub city level monthly catchment area meeting to evaluate the quality and performance of overall program, once each sub city monitor their activities independently, then after the health cadres take the QI activities independently and monitor the progress review on monthly bases on the regional program specific review meeting, which is a prove of sustainability. 


\section{Discussion}

This study was designed to assess the effect of Quality Improvement (QI) on achieving routine HIV viral load implementation in Addis Ababa, Ethiopia from January 2016 to December 2016 within the on hand budget-neutral, where the costs to make the changes are offset by the cost savings incurred by identifying opportunities to improve as Beck KL, it's a procedure by which improving processes and outcomes relevant to high-priority health needs, an organization reduces waste and costs associated with system failures and redundancy proactively without additional budget [13]. Sensitization training was conducted for laboratory personnel, health care providers and program management team at different time.

Quality Improve have been implemented at Addis Ababa health research Laboratory HIV viral load monitoring laboratory by which activities were routinely monitored, evaluated to check whether services offered by the laboratory were attest following the requirements of established guidelines and standard operating procedures of Ethiopian consolidated ART guideline and WHO using performance indicators on proposed change ideas adapting in to the existing scares budget devoid of entailing extra stab resource [4].

For a year we have followed, implementing QI with many up and downs and admired those that have tried to bring this quality improvement processes to the laboratory. After the significant remarkable achievement from QI, the facility endorses it and makes it capitalized to expand to other program and system in routinely manner [7].

Non-conformance performances of routine HIV viral load were managed by using a common QI tools used to diagnose quality problems of route cause analysis via fishbone diagram and also utilize a driver from the hypothetical frameworks address the factors deemed favorable for making QI in a sustainable, essential part of health services in Addis Ababa health system as James similarly proven [14].

This study depict the evidence that capacity building of professionals working on routine HIV viral load analysis, health care providers who are monitored HIV viral load status and program managers is the crucial part to bring the team on board proactively transform widely possess perceptions, inform low-cost and no-cost remedial actions, and improve the current work experienced, it is concordant with the finding of Leatherman et al. [15] where in order to boost up the adoption of quality improvement (QI) methods to improve healthcare quality in resource-poor settings, Healthcare leaders and improvement experts should incorporate their quality improvement to their routine activity plans by monitoring it in a regular schedule and exercises the feedback accordingly.

In the present study the run chart illustrate a staidly incremental of routine viral load performance in the regional HIV viral load laboratory after the implementation of quality improvement, which is concordance with a study done by Webster et al. [16] on quality improvement to accelerate highly active antiretroviral treatment coverage in South Africa, having a small increase in initiation rate during the start of the QI work in the PHCs and the HIV testing scale up.

The study attest that, the quality improvements implemented in Addis Ababa, turn out health care transformational impact with presented scares resources, without additional enquiry adds urgency to this schema, for the practicability of completing the schema in the near term is reinforced by evidence that quality improvements can be scaled up rapidly in other health system by establishing quality indicator for monitoring the performance by driving it via run chart, identified after the baseline assessment [17].
Likewise, health facilities should establish QI team, who are responsible and pioneer for reviewing and discussing QI findings and issues, and to jointly come up with improvement thoughts and way out for quality improvement in a dedicate manner with the respective stake holders, take a charge of be influence positively to accomplish improvement effectively and efficiently in the health system [18].

\section{Conclusion}

Quality Improvement is a game changer and it's a noble idea in transforming health Quality in order to achieving the third 90 of global ambitious target. The last 90 - it seems more striving especially in developing country, like Ethiopia having scares economical background and erratic and weak pharmaco-laboratory commodity management system. However, an organization that implements a QI model achieve and worth good experiences with a range of benefits: Improved patient clinical outcomes that involve both process outcomes and health outcomes. Improved efficiency of managerial and clinical processes. We strongly believes that healthcare has much to gain by successfully implementing quality improvement principles, it should be pinned and also all staffs should be embraces the philosophy of quality improvement in their routine programs.

According to the findings of this study, we winding up as quality improvement have a positive effect and it's the key driver to transform one activity, like on meeting the third 90 of global routine HIV viral load plan in Addis Ababa. Further study include suppression level of clients should be conducted.

\section{Limitation of the Study}

The following limitations should be into consideration, while readers inferring our results. Due to administrative and issues of access of quality of data, this study was conducted only in Addis Ababa city administration public health facilities (Regional reference laboratory and its refereeing facilities) plus viral suppression level of the individuals did not analyzed. Hence, the result would be more informative if more health facilities outside Addis Ababa and privates were included and further study include suppression level of clients should be incorporated.

\section{Ethical Approval and Consent to Participant}

Ethical clearance was obtained from Addis Ababa public health research and emergency management core process ethics and research committee .Confidential identifier was used to code data not mentioning participant's identities and their sites. Results and any information regarding patients' were kept confidential during and after the study/analysis.

\section{Availability of Data and Material}

The dataset analyzed during the current study, available from the corresponding author on reasonable request.

\section{Author Contributions}

Abay sisay, Bekelech Bayou and Abrham Tesfaye conceived and designed the study and collected data, performed analysis, interpretation of data and also critically reviewed of this manuscript.

\section{Acknowledgement}

First of all we would like to thank to our almighty GOD who giving us good health throughout our life. We also thank Addis Ababa Public Health Research and Emergency Management Core Process. Our last but not least gratitude goes to our families and friends for all the support and understanding during this study. 
Citation: Sisay A, Bayou B, Tesfaye A (2018) Quality Improvement (QI): A Splendid Driver for Achieving the Third 90 in Addis Ababa, Ethiopia. J AIDS Clin Res 9: 765. doi: 10.4172/2155-6113.1000765

Page 6 of 6

\section{References}

1. Federal HIVIAIDS Prevention and Control Office. HIVIAIDS strategic plan 2015-2020 in an investment case approach. Addis Ababa, Ethiopia.

2. Lundgren JD, Mocroft A, Gatell JM, Ledergerberg B, Monforte A, et al. (2002) A clinically prognostic scoring system for patients receiving highly active antiretroviral therapy: Results from the EuroSIDA study. J Infect Dis 185: $178-187$

3. Implementing HIV VL testing, WHO (2014) 90-90-90: An ambitious treatment target to help end the AIDS epidemic. Joint United Nations Programme on HIVI AIDS.

4. http://www.who.int/hiv/pub/amds/access-arv-2014/en/

5. https://www.hrsa.gov/sites/default/files/quality/toolbox/pdfs/qualityimprovement.pdf

6. Gilks CF, Crowley S, Ekpini R, Gove S, Perriens J, et al. (2006) The WHO public-health approach to antiretroviral treatment against HIV in resourcelimited settings. Lancet 368: 505-510.

7. https://www.globalfinancingfacility.org/sites/gff_new/files/Ethiopia-healthsystem-transformation-plan.pdf

8. Ethiopian Public Health Institute (2015) HIV related sub national estimates and projection target from year 2015-2019. EPHI, Addis Ababa, Ethiopia.

9. Central Statistics Agency (2008) Summary and Statistical report of the 2007 population and housing census results. Central Statistical Agency, Addis Ababa Ethiopia
10. http://article.sciencepublishinggroup.com/pdf/10.11648.j.ajlm.20160102.12.pdf

11. https://www.itacaddis.org/docs/2017_11_10_09_48_31_ARM\%202017. compressed.pdf

12. http://www.enao-eth.org/facility+detail.php?id=146

13. Beck KL, Larrabee JH (1995) A simultaneous analysis of nursing care quality and cost. J Nurs Care Qual 9: 63-71.

14. James $H$ (2014) The use of modern quality improvement approaches to strengthen African health systems: A 5 year agenda. Int J Qual Health Care 26: $117-123$

15. Leatherman S, Ferris TG, Berwick D, Omaswa F, Crisp N (2010) The role of quality improvement in strengthening health systems in developing countries. Int J Qual Healthc 22: 237-243.

16. Webster PD, Sibanyoni M, Malekutu D, Mate KS, Venter WD, et al. (2012) Using quality improvement to accelerate highly active antiretroviral treatment coverage in South Africa. BMJ Qual Saf 21: 315e324.

17. Hermida J, Salas B, Sloan NL (2012) Sustainable scale-up of active management of the third stage of labor for prevention of postpartum hemorrhage in Ecuador. Int J Gynaecol Obstet 117: 278-282.

18. Singh K, Brodish P, Speizer I, Barker P, Issac AE, et al (2016) Can a quality improvement project impact maternal and child health outcomes at scale in northern Ghana? Health Res Policy Syst 14: 45 